J3eA, Journal sur l'enseignement des sciences et technologies de l'information et des systèmes,

Volume 3, Hors-Série 1, 19 (2004)

DOI : http://dx.doi.org/10.1051/bib-j3ea:2004619

(C) EDP Sciences, 2004

Aide au paramétrage d'implant cochléaire par algorithme évolutionnaire

C. Bourgeois-République ${ }^{1}$ et P. Collet $^{2}$

${ }^{1}$ LERSIA

Université de Bourgogne

9 avenue A. Savary

BP 47870

F-21078 Dijon CEDEX, France

${ }^{2}$ Laboratoire d'Informatique du Littoral

BP 719

F-62100 Calais CEDEX, France

bourgeois.republique@free.fr, pierre.collet@univ-littoral.fr 


\title{
Aide au paramétrage d'implant cochléaire par algorithme évolutionnaire
}

\author{
Claire Bourgeois- République $^{1}$ et Pierre Collet $^{2}$ \\ ${ }^{1}$ LERSIA - Université de Bourgogne \\ 9 avenue A. Savary, B.P. 47870 Dijon cedex, France \\ ${ }^{2}$ Laboratoire d'Informatique du Littoral \\ BP719, 62100 Calais Cedex, France \\ bourgeois.republique@free.fr, pierre.collet@univ-littoral.fr
}

\begin{abstract}
Résumé - Ce papier présente une méthode basée sur l'utilisation d'algorithmes évolutionnaires et sur l'expertise médicale ayant pour but de régler automatiquement les paramètres déterminants des implants cochléaires (IC). Ce travail de recherche est réalise en collaboration avec le fabricant d'implants cochléaires MXM. Un Personal Data Assistant (PDA) est mis à disposition du patient pour qu'il puisse procéder de façon autonome au réglage de son implant, dans l'environnement sonore qu'il souhaite et qu'il est souvent difficile de recréer dans la salle de consultation du praticien.
\end{abstract}

Mots clés : implant cochléaire, PDA, algorithmes évolutionnaires, système expert, EASEA, réseau sans fil.

\section{INTRODUCTION}

L'implant cochléaire (IC) constitue une solution thérapeutique efficace chez les personnes atteintes de surdité profonde ou totale et qui ne tirent aucun bénéfice de prothèses auditives conventionnelles.

Il permet de rétablir une forme d'audition qui améliore beaucoup les capacités de communication, même si son efficacité est variable d'un patient à l'autre. En fait, elle dépend d'une interaction entre de nombreux paramètres parmi lesquels le réglage de l'implant cochléaire. Il est en effet difficile pour le praticien de trouver un réglage optimal du fait de la disparité des patients, de la difficulté qu'éprouvent les patients à interpréter leurs sensations mais aussi du fait du grand nombre de paramètres à optimiser.

L'objectif du travail décrit dans cet article est de proposer un logiciel interactif capable d'assister le praticien et le patient dans la phase de paramétrage de l'IC. Pour cela, une méthode basée sur l'utilisation des algorithmes évolutionnaires et sur l'intégration des connaissances d'un expert est proposée. Une partie du réglage peut se faire in situ en l'absence du praticien, par l'intermédiaire d'un PDA qui contient le logiciel de paramétrage automatique, basé sur une optimisation par algorithme évolutionnaire. Cet article présente successivement les paramètres pris en compte pour le réglage de l'IC et la classification adoptée. Une méthode d'optimisation stochastique est ensuite décrite et son choix est expliqué. La procédure de réglage est détaillée, à la suite de quoi les avantages de la solution proposée sont soulignés.

\section{PARAMETRES PRIS EN COMPTE DANS LE REGLAGE D’UN IC}

L'objectif des réglages est d'optimiser l'information délivrée par l'implant par rapport aux capacités électrophysiologiques des voies auditives du patient [4]. Il s'agit de privilégier les éléments pertinents contenus dans la parole, de manière à permettre une meilleure reconnaissance de celle-ci. Une cinquantaine de paramètres sont pris en compte lors du réglage. Il sont classés selon deux catégories : les paramètres déterminants et les paramètres d'affinage.

\section{A. Paramètres déterminants}

Ils influent sur le réglage de façon déterminante : la modification de la valeur d'un de ces paramètre peut modifier radicalement la perception du patient. On dénombre trois paramètres déterminants pour chacune des 15 électrodes de l'implant MXM :

- activation de l'électrode,

- offset,

- largeur de bande.

Pour chaque électrode, le paramètre d'activation est de type booléen. Ensuite, l'offset et la largeur de bande sont des paramètres de type entier, ayant des valeurs comprises respectivement dans les intervalles [549; 7263] et $[122 ; 7324]$.

On vient donc de dénombrer 45 paramètres dont 30 peuvent prendre de nombreuses valeurs différentes, même si l'expertise du praticien permet en pratique d'en restreindre en partie l'éventail.

\section{B. Paramètres d'affinage}

Ils permettent d'améliorer sensiblement le réglage obtenu à l'aide des paramètres déterminants, sans toutefois changer la perception du patient du tout au tout. On en dénombre cinq :

- volume du microphone,

- intensité,

- énergie d'adaptation,

- gain,

- sensibilité. 


\section{METHODE D’OPTIMISATION}

Le réglage de l'IC est appréhendé comme un problème d'optimisation.

La résolution d'un problème d'optimisation consiste à explorer un espace de recherche afin de maximiser (ou minimiser) une fonction donnée que l'on nomme fonction objectif [2]. Dans le cas présent, l'espace de recherche correspond à l'ensemble des solutions potentielles déterminées par les valeurs des différents paramètres et la fonction objective n'est autre que la qualité de perception du patient. On est en présence d'un problème inverse d'optimisation combinatoire non linéaire, qui présente de nombreux optima locaux.

Ces particularités, ajoutées à l'étendue de l'espace de recherche et à l'inexistance d'expression mathématique modélisant la fonction à optimiser rendent le problème d'optimisation proche d'un problème NP-complet, où la seule stratégie connue pour trouver l'optimum global consiste à tester méthodiquement toutes les combinaisons de valeurs possibles pour chacun des paramètres.

En effet, suivant le passé du patient (surdité pré ou postlinguale, d'origine traumatique, congénitale ou pathologique, nombre d'années passées entre l'apparition de la surdité et l'implantation, ... .), la profondeur de l'insertion de l'électrode, les neurones auditifs déficients, le réseau neuronal de traitement de l'information réagira très différemment aux impulsions électriques délivrées par les électrodes de l'implant cochléaire, ce qui rend chaque patient unique, minimisant par la même occasion la notion d'expertise du praticien.

Dans ces cas o`u aucune heuristique n'est vraiment valide, des méthodes d'optimisation stochastiques sont envisageables parmi lesquels les algorithmes évolutionnaires, qui ont pour particularité de largement explorer l'espace de recherche.

\section{ALGORITHMES EVOLUTIONNAIRES AEs}

Les AEs s'inspirent de la théorie développée par Charles Darwin au cours du 19ème siècle sur l'évolution génétique des espèces. L'intérêt porté à ces algorithmes vient du fait que la vision de l'évolution définie par Darwin peut être appréhendée comme un problème d'optimisation. En effet, l'idée d'auto adaptation sousjacente à cette théorie est que, sous l'influence de conditions extérieures, les êtres vivants évoluent progressivement au cours du temps vers des générations mieux adaptées aux conditions complexes de leur environnement.

Les AEs sont une classe d'algorithmes d'optimisation par recherche probabiliste. Ils reposent sur l'évolution d'une population de solutions potentielles qui, sous l'action de règles précises, optimisent un comportement donné exprimé sous forme d'une fonction, dite de fitness ou d'adaptation à l'environnement [3]. Le vocabulaire utilisé dans les AEs s'inspire grandement de la biologie et de la génétique.

\section{A. Principe des Aes}

Dans un premier temps, il faut définir le génome de chaque individu c'est-à-dire, dans le cas présent, les paramètres déterminants de l'implant cochléaire :

1. Une population d'individus est créée aléatoirement en s'inspirant toutefois de l'expérience du praticien pour mettre de côté les valeurs incohérentes.

2. La population est évaluée par le patient, ce qui donne aux individus le statut de parents.

3. Une sélection de parents est effectuée, basée sur la note donnée par le patient, dans le but de favoriser les meilleurs individus en vue d'une "reproduction," qui est simulée par deux opérateurs stochastiques [2] :

la recombinaison (ou croisement) qui à pour objectif d' échanger les informations entre plusieurs individus, ce qui doit permettre d'explorer l'espace de recherche,

la mutation qui, doit introduire de nouvelles informations en perturbant les individus, pour assurer la diversité génétique, c'est-à-dire la garantie d'une large exploration de l'espace de recherche, en quête du bassin d'attraction de l'optimum global. De cette fa,con, de génération en génération, les meilleurs gènes se propagent dans la population, en se combinant ou échangeant les meilleurs traits.

4. On reboucle ensuite sur l'étape 2 , jusqu'à-ce que le patient soit satisfait, ou jusqu'à-ce qu'il soit fatigué.

\section{B. Solution proposée}

Comme cela a déjà été suggéré, l'aide principale qu'apportera la méthode d'optimisation ne s'appliquera pas sur les paramètres d'affinages, o'u l'expert trouvera en quelques minutes un réglage optimal grâce à son expérience des réactions du patient, mais sur les paramètres déterminants qui sont difficiles à trouver.

L'algorithme évolutionnaire a été implémenté et testé, en attendant que la société MXM [7] réalise l'interface permettant au logiciel de paramétrer directement l'implant cochléaire d'après les individus à évaluer.

\section{PROCEDURE DE REGLAGE DE L'IC}

Le logiciel évolutionnaire, implanté sur le PDA en langage EASEA [1] est peu gourmand en ressources informatiques, ce qui signifie qu'il est possible de le faire s'exécuter sur un PDA actuel. Cette fonctionnalité est très importante, car il est très difficile de recréer dans le bureau du praticien l'ambiance bruyante d'un restaurant ou d'un hall de gare qui sont typiquement les cas où les patients éprouvent des difficultés de compréhension.

De ce fait, la procédure complète de réglage de l'implant est prévue de la façon suivante :

1. Lors de la première séance de réglage après implantation, le praticien connecte l'IC sur son microordinateur, et procède aux tests psychophysiques permettant de déterminer les seuils minimaux et de confort acceptables pour chaque électrode.

2. Le praticien configure le logiciel évolutionnaire dans le PDA avec les mesures effectuées à l'étape 1 . 
3. Le praticien et le patient lancent un réglage automatique à l'aide du PDA,

4. le patient teste et évalue le nouveau réglage à l'aide du PDA. Il attribue une note entre 1 et 10 et évalue la qualité du réglage à l'aide de huit appréciations qualitatives (résonne, sourd, criard, ....).

5. le patient évalue de nouveaux réglages avec le PDA jusqu'à satisfaction.

6. le praticien charge le réglage ayant obtenu la meilleure note sur son micro-ordinateur (sauvegarde) et procède à l'optimisation des paramètres d'affinage.

Par la suite, le patient pourra partir avec le PDA en prêt pour effectuer lui-même des réglages automatiques en situation, et les réglages seront sauvegardés à son retour à l'hôpital.

\section{BASE DE DONNEES}

Les réglages accompagnés des évaluations ainsi que les informations relatives aux patients alimentent une base de données gérée par le praticien.

La base de donnée est réalisée avec le logiciel Access pour raisons de compatibilité avec le système d'exploitation installé sur le matériel de l'hôpital.

L'intérêt de cette base de données est d'établir des statistiques, d'effectuer des requêtes afin d'élargir les connaissances des experts (médecins, chirurgiens, orthophonistes ....). L'étude des différents réglages et leur évaluation effectuée grâce à cette base de données permettra également d'ajuster l'algorithme évolutionnaire (valeurs des probabilités, opérateurs de recombinaison et de mutation. ..).

\section{LE PDA : INTERFACE MOBILE ENTRE PATIENT ET L'IC}

Pour rendre le patient le plus autonome possible dans le réglage de son IC et pour qu'il puisse procéder aux tests dans son environnement quotidien, une interface graphique a été développée sur un PDA léger sur lequel le patient pourra lancer un réglage et procéder à son évaluation.

L'option du PDA a été choisie car les machines actuelles possèdent de bonnes performances : écran haute résolution de $320 \times 320$ et 65536 couleurs, puissance de 400 Mhz, 64 Mo de Ram et 16 Mo de mémoire flash. ... Les fonctionnalités des réseaux sans .1 sont intégrées et permettent des connexions aisées avec le micro-ordinateur du praticien grâce aux protocoles 802.11 (wifi) [8] et bluetooth [6].

Le système d'exploitation utilisé est windows CE, et le code du logiciel d'optimisation et les données sont stockées dans le PDA [5]. Lorsque le patient revient voir le praticien après plusieurs jours, le PDA se connecte au PC par l'intermédiaire du réseau Bluetooth, pour rapatrier les réglages effectués.

Le débit faible de Bluetooth comparé à Wifi n'est pas handicapant, car il s'agit simplement de transmettre des paramètres et des évaluations. La programmation prévoit une identification du patient afin d'éviter qu'un patient ne télécharge ses paramétrages dans le dossier d'un autre patient.

\section{CONCLUSION ET PERSPECTIVES}

Le module de paramétrage de l'implant par le PDA n'étant malheureusement toujours pas disponible au moment de la rédaction de cet article, les tests physiques n'ont pas encore pu avoir lieu.

Cependant, une séance de 90 minutes chez le praticien ne permettant de tester que 5 à 6 réglages au plus, il est certain que la procédure décrite permettra d'explorer plus largement l'espace de recherche et de proposer des réglages que le praticien n'aurait jamais soumis. Le paramétrage est très rapide, (le transfert des nouveaux réglages dans le processeur de l'implant ne prend que quelques secondes) et permet au patient d'être plus autonome en lui donnant la possibilité de régler son implant dans des conditions non reproductibles dans le cabinet du praticien.

\section{RÉFÉRENCES}

[1] P. Collet, E. Lutton, M. Schoenauer, J. Louchet, Take it EASEA ,Proceedings of the 6th Conference on Parallel Problem Solving from Nature, PPSN VI, Eds. M. Schoenauer et al., Springer Verlag, LNCS 1917, pp 891901, September 2000, Paris, URL :

http ://easea.sourceforge.net/.

[2] Fogel, D.B., Evolutionary Computation: Toward a new Philosophy of Machine Intelligence, IEEE Press, Ed. J.B. Anderson, NY 1995.

[3] D. E. Goldberg, Genetic Algorithms in Search, Optimisation and Machine Learning, 1989, Addison Wesley.

[4] Loizou, P., Poroy, O., Dorman, M., The e.ect of parametric variations of cochlear implant processors on speech understanding, Journal of Acoustical Society of America , 108(2), 790-802 2000.

[5] R. Litiu and A. Prakash, Developing Adaptive Groupware Applications Using a Mobile Component Framework, proceedings of the ACM 2000 Conference on Computer Supported Cooperative Work, Philadelphia, PA, December 2000.

[6] Marzegalli, L., M. Masa, M., Vitiello, M., Adaptative RTP/UDP/IP Header Compression over Bluetooth, European Wireless 2002, Florence, Italy 2002.

[7] MXM laboratories, URL : http ://www.mxmlab.com/. [8] S. Sheu, J. Chen, H. Tseng, H. Chiang, A Safe Multiple Access-Rates Transmission Scheme for IEEE 802.11 Wireless Networks, in Proc. 2003 Int. Conf. Advanced Info. Networking Appl., Xian, China, March 2003. 